\title{
The Application of Mobile Big Data in City Planning Area
}

\author{
Xia Xiaotang \\ Wuhan University of Science and Technology \\ College of Urban Construction \\ Wuhan, China \\ xiaxiaotang2010@163.com
}

\begin{abstract}
With the development of the city, transportation becomes an important factor in the development of a city. Starting from the drawbacks of the traditional means of solving the traffic information collection, using mobile phones as the signal collection of traffic information means the emerging mining and using the valuable information in the era of big data, which brings new ideas for the traffic policy and services for the collection and analysis of $O D$ to solve the traffic problems in urban planning.
\end{abstract}

Keywords-Mobile signal; traffic planning; OD collection

\section{INTRODUCTION}

With the rapid growth of population and urban modernization, the urban transportation is facing severe challenges, promoting the sustainable development of urban transportation system by adopting a variety of measures. In the process of rapid development, due to the limitations of traditional traffic technology, to achieve better use of a variety of information resources to get a fine, quantitative, scientific analysis to achieve the goal of traffic congestion control. Therefore, it is urgent to provide timely, high accuracy, high reliability data support for urban transportation planning, construction and operation management. In 2015, the 13th Five-Year plan indicates that, staff travel feature data is the key basic data, and the basis of quantitative analysis of the decision-making process in city traffic planning, and traffic planning is the priority among priorities of the whole traffic system construction ${ }^{[1]}$. The traditional traffic information collection technology has been ignored, while the traditional means of traffic collection is large, complex and inaccurate.

Affected by the popularization of the Internet and the impact of the rapid development of information technology, networking, cloud computing and other information technologies, many changes have taken place in the study of traditional urban planning. Focusing on the micro subject Urban residents' demand for urban development has become the hotspots of urban planning research. The big data method and technology will become an important means to support this hot research ${ }^{1-2]}$. Discovering, mining, processing, and analyzing the big data which obtained by the process of urban development can provide new ideas and methods for urban planning. Using big data thinking mode, methods and techniques, can accurately, quantitatively and accurately analyze and study urban problems, can guarantee the planning program more scientific, can make urban infrastructure, public service facilities layout more reasonable and efficient, can make public participation in planning more convenient and directly, and make planning decisions more democratic

With the popularity of mobile phones, the promotion of $4 \mathrm{G}$ networks, the use of the increasing rate of travel groups in the proportion of mobile phone users are also risen. At the same time, the daily mobile phone network generating mobile signaling data is also rising. The emergence of mobile phone, FCD, IC card collection staff travel feature information technology are several news mobile data acquisition means, are valuable information to mining, can reveal more regularities, bring new ideas for ladder transportation decision and service. Urban planning and management is an accumulatingdisseminating process of a lot of spatial information. With the acceleration of urbanization, the spatial information serving the planning and management has been quickly developed, and the basic geographic data, planning approval data and socioeconomic data have formed the huge information flow. The information is various in different aspects, levels and types, and there are information supporting urban planning or produced by planning, covering and the science and specialty. These multi-type, multi-temporal and multi-resolution graphics, images, text and other heterogeneous data are organically integrated to construct the urban planning database, forming the information resources that can provide urban planning with the qualitative, quantitative, positioning analysis and aid decision-making, which can record and reflect the urban history, status quo and planning, and easily and promptly update the data to achieve mass storage, efficient management, continuous updating and good service, provide important database resources for the construction of "digital city" and provide detailed geographical criteria for the society, government and relevant departments.

\section{The DeVELOPMENT Form OF URBAN PLANNING IN THE ERA OF BIG DATA.}

\section{A. The characteristics of big data era in urban planning}

In the process of the traditional city planning, the analysis of the urban space is the use of questionnaires, interviews, etc. With the continuous development of information technology, the era of big data, network, mobile phone, data cards and other rapid development, in such circumstances, opportunities and 
new challenges to the city space planning also brought. Its main features are as follows: first, the use of data in the era of big data processing of urban space planning, the use of the system to understand the positioning of urban space. Second, the era of big data has a large number of capacity, the actual situation and characteristics, and the extraction of geographic information features, and the analysis and research of urban space in China ${ }^{[3]}$.

\section{B. The development of the era of big data in the process of urban planning}

In recent years, in the process of urban planning, it is mainly based on the analysis and research of urban space geography in our country. From the perspective of urban micro analysis, people in daily activities and life. To some extent, it determines the distribution of urban space in china. The process and development in the era of big data, the global positioning system, web logs, social interests, mobile phone data and floating car data and data bus card etc., are analyzed and studied further. This will not only help the relevant staff in the process of urban spatial planning, but also provides a more intuitive result. The planning of urban space can be more detailed analysis and research. At the same time, it can also be a certain degree of analysis of the affordability of the city, to the greatest extent to protect the reasonable distribution of urban space $^{[4]}$.

\section{The Application Of Big DATA ERA IN Urban PlanNing.}

From the point of view of urban planning, the era of big data only has a wealth of data, forms, etc., but also a certain change in urban planning, breaking the traditional pattern of urban planning. With the continuous development of the times, information technology has become the mainstream of the development of the times, thus promoting the development of the era of big data. And it has been applied in various fields of our country. In the process of urban planning, the effective application of the big data era to urban planning, the following is a brief analysis of the use of big data era in the process of urban planning:

\section{A. From the traditional urban planning to the era of big data change}

In the city planning in the traditional process, is often too dependent on statistics, questionnaire survey, literature research and analysis of the form, so in the process of city space planning, due to the form of backward. There are many problems. But in the era of big data in the process of city space planning, the use of information technology and other forms of treatment to a certain extent on the relevant data, and in-depth analysis and study of the relevant data, thus to our country city planning to conduct a comprehensive analysis, and to plan the city's comprehensive show in people's eyes. The staff in the use of big data era of the city planning process analyzes and studies the use of massive, multi-source, spatiotemporal data form, especially the analysis and Research on social media data, mobile phone data and sensor, which can effectively help the staff of city planning, space and time. The city planning process in the era of big data, but also to me. To a certain extent, the economic development and transportation of the country are analyzed and studied. In the era of big data development, the use of small sample data, processing data on city planning, it is not only to the staff to show more intuitive data, but also provides an important reference for the city planning.

\section{B. In line with the trend of the times}

In the process of traditional urban planning, due to the use of the form is too old, did not keep up with our country. On behalf of the development of the trend, to a certain extent, the urban planning has formed a certain lag. In fact, the lag refers to China in the traditional city planning process, due to a single technical means, resulting in the processing and analysis of relevant data, to a certain extent caused by the city of space planning not only play a role, but will play a role of anti. This will hinder the development of China's urban economy to some extent. However, in the process of development in the era of big data, the use of advanced information technology, the related data were processed and analyzed in a timely manner, and in the analysis and processing process, the corresponding process is simplified, this can effectively avoid the disadvantages of the traditional city planning ${ }^{[5]}$.

\section{Improve work efficiency}

The traditional urban planning is relatively simple in form, and the method is backward. In the process, it is often used to collect, analyze study and deal with the related data. In this case, not only can't guarantee the accuracy of the relevant data, the city space planning did not play a good effect, but also increase the workload of the staff. In the context of the era of big data, the use of information technology, the relevant data collection, analysis. A series of work flow, such as research, processing, and the use of a rich database form, the city space to a certain extent, the accuracy of the relevant data. This not only ensures the accuracy of the relevant data, but also plays an important role in urban spatial planning in China.

\section{URBAN GENERAL PlanNING AND THE APPLICATION OF URBAN REGIONAL TRANSPORTATION PLANNING SUBSYSTEM}

\section{A. Analysis of urban spatial structure}

The urban-rural spatial axially moves along the urban development axis. According to the purpose of the trip (based on family, work place, etc.), statistics out the OD traffic exchange between two regions, on different types of date (from Monday to weekend and holidays), in different range of time (morning and evening peak, flat peak), to determine the extent of the link between the two regions. At the same time, it can also be used to analyze the axial movement of urban and rural space along the axis of urban development, which is the basis of urban general planning and regional planning.

Analysis on the evolution of urban spatial structure. Through the year residents travel data accumulated, summed up the characteristics and rules of city traffic development, is conducive to a better understanding of the whole city, and the impact on the traffic in the city evolution and future development trend has better judgment, is an important data base for overall planning and revision of the city ${ }^{[6]}$. 


\section{B. Travel in the area and its correlation with the surrounding} area

In certain areas, such as administrative district, separate statistics were in the region, range, determine the strength associated with the surrounding area of the current area, which is relatively independent in the city, or as a center of regional or sub center area. Such data can be used as an important basis for urban agglomeration planning and urban general planning.

\section{Contact with the surrounding city passenger flow}

With the help of mobile phone users to identify the path of access to the city, as well as the attribution information of mobile phone users, the analysis of the current situation of the city and other cities in different time range of the passenger flow. Such data is one of the important bases of the airport, railway station and other external hub planning, as well as an important basis for the planning and design of World Expo, World Horticultural Exposition and other large-scale activities.

\section{CONTACT WITH THE SURROUNDING City PASSENGER FlOW}

\section{A. Key channel planning and optimization}

Long term historical data mining, grasp of the characteristics of the current situation of rail passenger travel provides scientific data support for rail network travel model and the network ${ }^{[7]}$.

Population distribution along the track. The distribution of the population along the track is important reference information for the planning and evaluation of railway track and the surrounding traffic. Timely understanding of the distribution of the population and the working population of the rail line can provide scientific and detailed data support for the adjustment of the rail transit network and the future planning of the traffic network.

Traffic generation and traffic attraction along track. The use of mobile phone positioning data can also be used to identify rail transit passenger rail route and transfer station, according to the spatial distribution of the source and destination of rail transit passengers, this paper analyzes the service radius, service orientation, etc., as one of the basis of rail transit lines, site planning and layout.

Service area along track. According to different site intensity of land development and utilization, determining the rail transit station in the service range of radiation in different directions, can be regarded as important basis for rail transit line selection or site location, site of interval set.

\section{B. Public transportation network optimization}

In order to meet the practical needs of the improvement of bus optimization, we should carry out joint analysis work, mobile phone data bus IC card data, GPS data bus, with a comprehensive and systematic grasp of the overall residents travel demand and public transportation demand and service level, by comparing the multi dimension analysis for public transit routes, stations, transport capacity adjustment with the construction of public transportation, the development of strategic planning provide quantitative analysis methods and data support ${ }^{[8]}$.

\section{Application of Urban Traffic Model Data Docking}

\section{A. Parameter calibration of urban traffic planning model}

Calibration of traffic generation coefficient and traffic attraction coefficient under different land use characteristics, parameter calibration of OD gravity model, parameter calibration of inter-regional traffic mode, parameter calibration of traffic assignment impedance function, and population growth rate, travel distance and time parameters, etc, all can provide reliable data support for parameter calibration of urban traffic planning model.

\section{B. Data checking of urban transportation planning model}

Based on the analysis of mobile phone data, for the audit line segment or distinguish the specific channel, to get the traffic changes across the audit line on both sides of the situation, may be used in checking for data distribution OD traffic planning model, such as the use of mobile phone data analysis on both sides of the passenger traffic check line. In the face of a particularly large number of channels crossing the check line, usually the traditional survey cannot be used. Mobile data break the barriers of the region and achieve effective access to large channel passenger flow changes.

\section{APPLICATION OF URBAN TRAFFIC IMPACT ANALYSIS}

\section{A. Evaluation of regional passenger flow characteristics}

In the development and construction of large projects, flexible use of information analysis results of multi-source data to analyze the current situation of the project can provide reliable and accurate data basis for traffic forecast and improvement measures in traffic impact analysis ${ }^{[8]}$.

Take Zhuhai as an example, in 2015 the city is conducting an informational pilot project of traffic impact analysis, intends to use the mobile phone data, GPS data bus, taxi GPS and IC card data, providing regional travel amount, taxi trips, bus travel data of traffic impact analysis of pilot project, get traffic status of multiple modes of transportation, and provide a reliable basis for future traffic forecast. At the same time, using the mobile phone data to analyze the project area to a few large areas of travel volume and proportion, used to study the traffic distribution of the traffic trip project, is the basis of traffic assignment and traffic flow superposition. In addition, through the mobile phone data combined with the nature of the land, the peak hour traffic rate of the analysis unit is obtained, which provides a reasonable and reliable data reference for the peak hour traffic rate of the buildings.

\section{B. Comparative analysis of regional passenger flow}

In the informational pilot project of traffic impact analysis in Zhuhai, the analysis results of mobile phone data also provide research projects surrounding the same nature of land plots of the peak hour traffic rate, and provide horizontal reference of the determination of traffic incidence in research projects. 


\section{SUMMARY AND PROSPECT}

The city is developing day by day, and the traffic development is also the key factor that affects the development of a city. The limitations of traditional traffic information collection methods force the birth of new technologies. With the rapid development of network and communication, mobile phone has become a necessity in people's daily life. In this paper, I use mobile phone signal data which plays an important role in transportation planning, based on the analysis of the OD spatial distribution of commuting travel demand to plan and optimize the key channel; furthermore, in the aspect of rail transportation, the population distribution along the track and the service range along the track can be planned and optimized.

In the future, we can continue to study in the city's overall planning and zoning, urban public transport network planning, traffic impact analysis and urban operations management.

\section{ACKNOWLEDGMENT}

The research is supported by the humanities and social science research project of Hubei Provincial Education Department (NO: 15Q032) and supported by the scientific research project of Hubei Provincial Education Department (NO: Q20141106). And supported by the scientific and technological youth backbone cultivation plan project of Wuhan University of Science and Technology(NO:250089).

\section{REFERENCES}

[1] Hu Ling, Liu Qiang.Application of ArcSDE Geodatabase to GIS Application of Urban Planning Management [J] .Computer Science, 2006, 33 (12): 125-127.

[2] Ye Yu, Wei Zongcai, and. Urban planning response in the era of big data [J]. Planners, 2014, (8): 5-11.

[3] Qin Xiao, Zhen Feng, Xiong Lifang, et al. Research methods of temporal spatial behavior in the era of big data [J]. Advances in geographical science, 2013, (9): 1352-1361.

[4] Xi Guangliang, Zhen Feng. Research on the urban planning innovation under the support of the process or the result of a large data [J],2015.(1):19-23.

[5] Jin Xianfeng, Zhang Zelie, Wang Chuanqi, Zhu Xiaoqing. Thinking on the construction of informatization in the era of big data [J]. Planners, 2015, (3): 135-139.

[6] Sui Mingming, Li Zonghua.Study on the realization of spatial data sharing and interoperability in planning land management [J]. Geospatial Information, 2005, 3 (3): 16-18.

[7] Lu Xinhai.Multi-source urban planning data fusion and integration method [J] Surveying and Spatial Geographic Information, 2005, 28 (4): 47-49.

[8] Li Heng, Yang Dan, Fang Weitao, et al. Design and research of workflow management system [J] .Computer Science, 2008, 35, (8): 293-296. 